\title{
A simulation of the coupled problem of magnetohydrodynamics and a free surface for liquid metals
}

\author{
S. Golak \& R. Przyłucki \\ Faculty of Materials Science and Metallurgy, \\ Department of Electrotechnology, Silesian University of Technology, \\ Poland
}

\begin{abstract}
The simulations of induction melting and stirring of the molten metal have focused on the flows inside the bath, while the meniscus forming on the surface of the bath has been neglected. The fact of ignoring the meniscus results in ignoring a whole range of problems caused by the phenomenon, and in consequence all quantitative analyses of the processes occurring on the metal-gas interface are significantly distorted because of the underestimation of the real free surface of the metal. A tendency to ignore the meniscus in the studies on the processes of induction melting results from the complexity of the problem. In order to correctly simulate the phenomenon we are forced to make allowances for a triple coupling of magnetic field, flow velocity field of the molten metal, and shape of the bath surface. The paper presents the methodology for conducting a simulation in which such allowances are made.
\end{abstract}

Keywords: magnetohydrodynamics, meniscus, induction melting and stirring.

\section{Introduction}

Today, the processes of induction melting and stirring are commonly used in metallurgy. At the same time constant development of the devices utilised in these processes can be observed. In the case of induction furnaces applied for metal melting the researchers focus on increasing their unit power, with the aim to reduce the charge melting time, which in turn means higher efficiency of the furnace. The increased power of the furnace results in enhanced electrodynamic 
field affecting the already molten metal. Consequently, it is hydrodynamic phenomena that acquire significance in the processes of induction melting. In effect, the research concerning the induction furnace design, which previously concentrated only on the analyses of electromagnetic and temperature fields, now must be extended to the issue of modeling the hydrodynamics of the metal bath.

So far it has usually been assumed in the research that the area of the liquid metal is determined after it has been melted. In such a case there is a possibility to run a simulation of the heating process of the liquid metal in two separate stages. The first step is to determine the distribution of electromagnetic forces affecting the metal, which can be done when metal geometry, materials parameters, inductor geometry, and supply parameters are known. With the assumption that the geometry of the liquid metal is fixed the obtained distribution of the field forces is constant, too. The second step includes hydrodynamic calculations for the known geometry of the metal, for the material parameters which can influence hydrodynamics, and for the distribution of forces determined in the first step (Adler and Schwarze [1]). Owing to this most widely used methodology, the problem is boiled down to a single-phase flow, which on the one hand simplifies the calculations but at the same time is far from being realistic.

In a real process of metal melting a convex meniscus occurs. It is initially dependent on the resultant of the surface energy of the crucible walls and the surface tension of the liquid metal which is depending on the liquid surface area. However, in case of induction melting the ultimate shape of the meniscus and the degree of the phenomenon are strongly influenced by hydrodynamics inside the metal induced by electromagnetic field. The main difficulty with such simulation of the induction melting process is the fact that the shape of the meniscus is affected (through the hydrodynamic phenomena caused by it) by electromagnetic field, and electromagnetic filed is in turn dependent on the geometry of the liquid metal. When one wants to run a simulation of the melting process taking into account the meniscus, the coupling between the shape of the metal bath and electromagnetic field must not be neglected. In this case the hydrodynamic simulation will concern a two-phase system of liquid metal-atmosphere.

Any neglect of the meniscus in induction melting will significantly distort the principal quantitative information concerning the process, that is the power output in the charge. Besides, the information about the real shape of the meniscus is of practical use for this process as it allows a prediction of the potential problems with the barrier protection of metal, oxidation of its surface and wearing off of the crucible lining.

A process similar to induction melting is induction stirring, which is often an immediate continuation of the former one. Both processes can also take place simultaneously. The goal of induction stirring is homogenizing of the liquid metal and supporting the processes occurring at the liquid metal-gas interface through providing suitable substrates near the area and transporting the products. An example of such a process might be elimination of impurities from the metal 
through evaporation, as it takes place in the process of removing lead from copper (Blacha et al. [2]).

Also in this process, neglecting the occurrence of the meniscus or imprecise estimation of its shape might distort the obtained results completely. First, it must be remembered that estimating the effectiveness of the metal homogenizing process is based on the determination of metal velocity field, which may be calculated correctly only in case of precisely assigned metal geometry and the distribution of electromagnetic forces. Ignoring the meniscus will determine the velocity field that is far from the real one. Still, this distortion may be seen as relatively small in comparison with the error made when estimating the phenomena occurring at the liquid metal-gas interface. The reason is practically linear dependence of the intensity of these phenomena (e.g. evaporation of lead from copper) on the surface area of contact between metal and gas. An assumption of an unreal shape of the metal surface may totally distort the quantitative measure of the reactions occurring on it.

For the above reasons it can be said that precise determination of the meniscus is essential for the accurate calculation of most quantitative measures representing utility values of induction furnaces and stirrers.

\section{Methodology}

The software available on the market enables to run the simulations of the induction melting and stirring processes in which the coupling between electromagnetic field and flow field is taken into account. Examples of such applications packages are Ansys Fluent MHD.

However, in case of induction melting and stirring velocities of the liquid metal are so small that their influence on the electromagnetic field can be ignored. That is why this model of coupling between metal hydrodynamics and electromagnetics is of no use in case of the devices discussed in this paper.

When analyzing the functioning of electromagnetic furnace and stirrer, the simulation performed should allow for the coupling among electromagnetic field, velocity field induced by it, and the changes in the shape of the liquid metal free surface caused by the metal flow. Unfortunately, to the authors' knowledge no commercial software available on the market allows such a possibility. Therefore it proved necessary to seek for another solution. The authors of this paper decided to create their own system on the basis of the existing software devoted to hydrodynamic and electromagnetic calculations. The main reason for this decision is a high level of development of these instruments, significant optimisation of their codes and their high efficiency, which are extremely difficult to obtain in one's own solutions created by a small team.

The operation of the induction furnace and stirrer was run in 2D space, which was possible thanks to the fact that the configuration considered here both from electromagnetic and hydrodynamic perspective can be regarded as axially symmetrical. The assumption was that the simulation started with the formation 
of the meniscus, whose shape was dependent only on the resultant of the surface energy of the crucible walls and the total surface tension of the liquid metal.

The first stage entailed the analysis of hydrostatic field through which the initial geometry of the liquid metal was determined. The meniscus shape was determined by VOF (volume of fluid) method allowing a simulation of behaviour of separate, immiscible phases (Hirt and Nichols [3]). In the study discussed here the interaction occurred between liquid metal phase, gas phase, and the crucible wall. The simulation yielded a set of points defining a curve that describes the shape of the free surface of the metal.

During the next stage of the simulation the obtained geometry was used to determine electromagnetic field. The result was the distribution of electromagnetic forces acting on the liquid metal.

The meshes applied in the programmes for electromagnetic and hydrodynamic calculations do not overlap. The main reason is different methods of solving the differential equations describing electromagnetic and hydrodynamic fields. In the former the finite element method is widely used, while in the latter the finite volume method is preferred because of greater universality. Additionally, since the electromagnetic and hydrodynamic phenomena are different from each other in nature, the optimal mesh for electromagnetic calculations is not optimal in case of hydrodynamic calculations.

For this reason the exchange of data between the programmes for electromagnetic and hydrodynamic calculations was based on a rectangular mesh of density twice as high as the density of the meshes used in electromagnetic and hydrodynamic simulations. The decision concerning such density was taken on the basis of Kotielnikow-Shannon law of signal sampling.

The values of the force-field components in the nodes of the mesh used for electromagnetic calculations were recalculated to the nodes of the rectangular mesh by bilinear interpolation.

The known distribution of electromagnetic forces made it possible to proceed to the simulation of liquid metal hydrodynamics aimed at the determination of the current distribution of liquid metal velocity and the change in the original shape of the meniscus caused by electromagnetic forces. Certainly, the rectangular mesh containing the distribution of forces had to be transformed into the node mesh applied in hydrodynamic calculations. Once more bilinear interpolation was used.

Unfortunately, the change in the shape of the meniscus induced by electromagnetic field causes some changes of the field itself. That is why it was necessary to go back to electromagnetic calculations in order to update the field distribution. The set of points defining the curvature of the free surface are the input data for the electromagnetic calculations programme.

The main problem encountered at this point is the rate of the above updating. The update rate of field distribution during the hydrodynamic simulation is closely connected with the communication rate between the two separate programmes and with the need to conduct relatively time-consuming electromagnetic calculations. 
The hydrodynamic simulation is run in the unsteady mode, meaning that it analyses the change in time. An important element is here to adjust an adequate time step. When VOF method is used to trace the free surface of the metal, a time step below $10^{-4}$ second is often required in order to ensure the convergence of solution. Since this is the updating rate of the free surface shape, the optimal solution would be to update the distribution of electromagnetic forces with the same frequency, that is 10000 times a second. However, the exchange of such amount of data between programmes and the necessity to conduct timeconsuming electromagnetic calculations make the whole issue very difficult because the simulation time of the stabilization of free surface shape may even be as long as a few dozen seconds. Total time of simulation would then become excessively time-consuming.

The only way out seems to be less frequent updating of the electromagnetic field distribution. In such a case another problem occurs, and namely the decision concerning the moment of calculating the forces. This decision may depend either on the selected time step or the change in the shape of free surface. Because the distribution of electromagnetic field does not depend directly on time but on the actual geometry of the metal, the method based on the monitoring of the surface shape seems to be better justified.

For the sake of the simulation a scalar measure was defined which reflects the change in the shape of free surface compared with the surface for which the previous electromagnetic calculations were conducted. Exceeding the threshold value (adjusted experimentally) means the necessity to repeat the calculations.

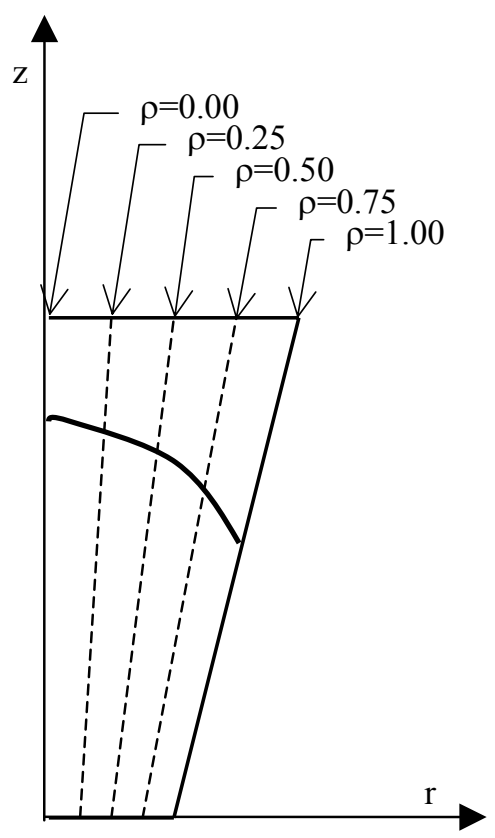

Figure 1: Relative radial coordinate. 
Before the definition of the above measure is provided first the notion of relative radial coordinate $\rho$ should be introduced. It denotes the ratio of the absolute coordinate $\mathrm{r}$ to the crucible radius $\mathrm{R}$ ratio at a given height, eqn. (1). Figure 1 shows graphic interpretation of the relative radial coordinator.

$$
\rho=\frac{r}{R(z)}
$$

where:

$r, z$ - radial and axial coordinates of the point

$R(z)$ - function representing the dependence of crucible inner radius on radial coordinate

On the basis of the measurements are obtained the dependencies of the distance of bath surface from the crucible bottom as function of relative radius for the original surface $H_{o}(\rho)$ and the current surface $H_{c}(\rho)$.

Knowing these values the measure of the surface change $S C$ can be determined:

$$
S C=\int_{o}^{1}\left|H_{o}(\rho)-H_{c}(\rho)\right|^{n} d \rho
$$

where:

$\rho$ - relative radial coordinate

$H_{o}(\rho)$ - dependency of the distance of original metal surface from the bottom in function of relative radial coordinate $\rho$

$H_{c}(\rho)$ - dependency of the distance of current metal surface from the bottom in function of relative radial coordinate $\rho$

$n$-empirical coefficient

Unfortunately, the experiment proved that the assumption that the distributions of electromagnetic forces between the consecutive updates are constant caused that the threshold value of the shape change measure had to be so low that the updating rate of the force field was only slightly lower than the time step in the hydrodynamic simulation. In any other case the convergence of solutions was not possible to obtain. This situation is easily explained by the physics of electromagnetic field, whose penetration is limited only to small distances from the upper, lower and side surfaces of the liquid metal. That is why the strongest electromagnetic forces occur in the upper and lower areas of the metal, nearby the crucible walls. Even the slightest shifts of the free surface cause significant changes in the distribution of the electromagnetic forces in its vicinity.

It proved necessary to apply at least an approximate extrapolation of the field distribution based on the changes in the bath shape between the consecutive updating steps. In the simulation considered a simple mathematical extrapolation was chosen. The process of extrapolation entails a comparison of the free surface curvature for which the electromagnetic calculations were conducted with the current curvature, and on the basis of this a re-scaling of the electromagnetic field determined for the original curvature, eqn. (3). 


$$
\vec{F}_{c}(\rho, z)=\vec{F}_{o}\left(\rho, \frac{H_{o}(\rho)}{H_{c}(\rho)} \cdot z\right)
$$

where:

$\vec{F}_{c}(\rho, z)$ - extrapolated vector of electromagnetic force at the point with the coordinates $(\rho, z)$

$\vec{F}_{o}(\rho, z)$ - vector of electromagnetic force at the point with the coordinates $(\rho, z)$ calculated from the magnetic field equations

$H_{c}(\rho), H_{o}(\rho)$ - dependency of the distance of current and original metal surface from the bottom in function of relative radial coordinate $\rho$

The above formula is a heuristic one and it is not derived from any physical equations of electromagnetic field. However, this strictly mathematical spatial transformation observes the rule that the highest values of electromagnetic forces are encountered at the boundaries of the metal charge, and it does not change their directions. Its application resulted in a surprisingly efficient hundredfold reduction of the frequency of electromagnetic calculations, limiting the total simulation time to an acceptable amount.

Figure 2 presents a diagram of the simulation methodology discussed here.

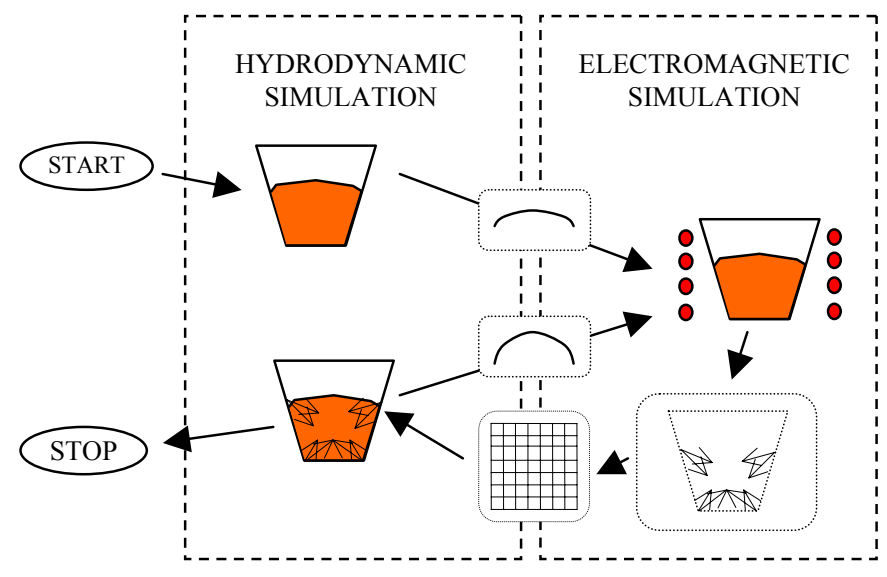

Figure 2: $\quad$ Schema of the method.

\section{Example}

An experimental simulation was run as part of the presentation of the method. The stirring process of the liquid aluminium was performed in a cone-shaped crucible in order to prove the high degree of the deformation of the liquid metal surface. Figure 3 presents a diagram of the modeled object. Table 1 presents the materials, supply and geometric parameters of the simulation. 
A 176 percent change was recorded in the free surface area of the liquid metal (table 2). It means that if the meniscus is not taken into account in the calculations, a similar degree of error should be expected in case of all quantitative analyses of the phenomena occurring at the metal-gas interface.

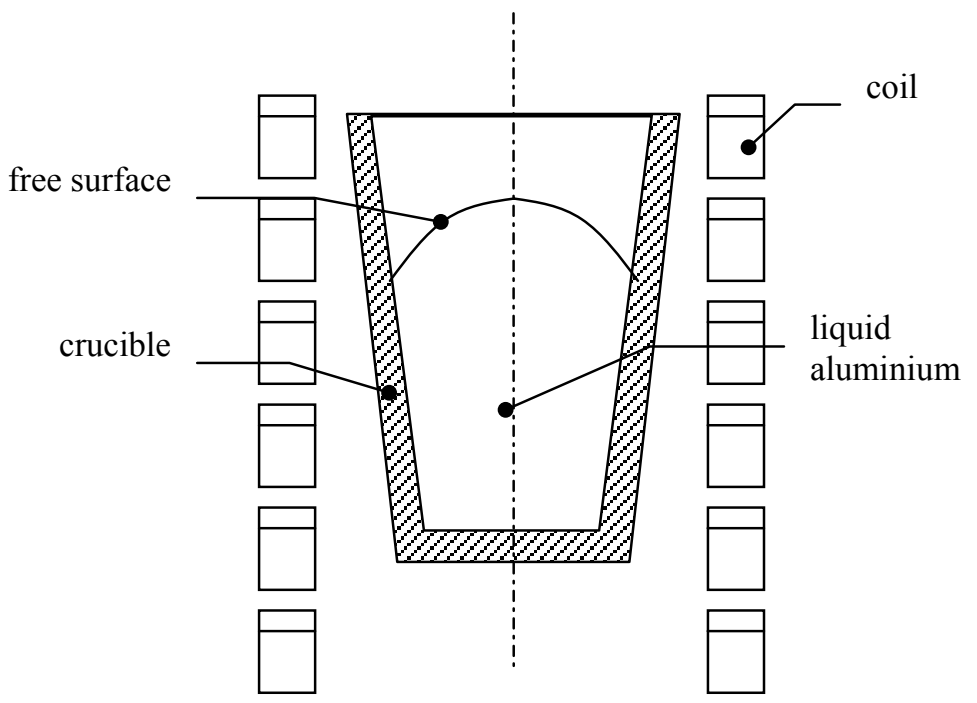

Figure 3: $\quad$ Modeled object.

Table 1: $\quad$ Parameters of the simulation.

\begin{tabular}{|c|c|}
\hline Quantity name & Value \\
\hline Frequency & $3 \mathrm{kHz}$ \\
\hline Current of source & $2903 \mathrm{~A}$ \\
\hline Resistivity of aluminium & $8.810-8 \Omega \mathrm{m}$ \\
\hline Volume of aluminium & $5.31 \cdot 10^{-4} \mathrm{~m}^{3}$ \\
\hline Density of aluminium & $2375 \mathrm{~kg} / \mathrm{m}^{3}$ \\
\hline Viscosity of aluminium & $9,5 \cdot 10^{-4} \mathrm{~kg} / \mathrm{m} \cdot \mathrm{s}$ \\
\hline Surface tension & $1.0 \mathrm{~N} / \mathrm{m}$ \\
\hline Contact angle & $120^{\circ}$ \\
\hline Height of crucible & $0.15 \mathrm{~m}$ \\
\hline Bottom radius of crucible & $0.036 \mathrm{~m}$ \\
\hline Top radius of crucible & $0.051 \mathrm{~m}$ \\
\hline
\end{tabular}

Table 2: $\quad$ Free surface area for different meniscus types.

\begin{tabular}{|c|c|}
\hline Meniscus & Free surface area $\left[\mathbf{m}^{2}\right]$ \\
\hline not taken into account & 0.006617 \\
\hline natural & 0.006675 \\
\hline intensified by electromagnetic field & 0.017046 \\
\hline
\end{tabular}


a)

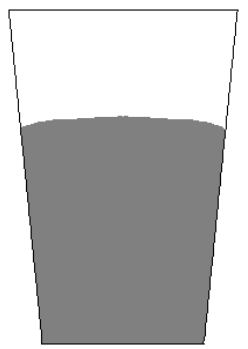

b)

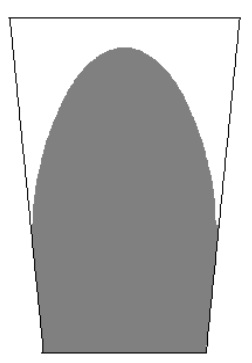

Figure 4: Geometry of the liquid aluminium: a) natural meniscus, b) meniscus intensified by electromagnetic field.

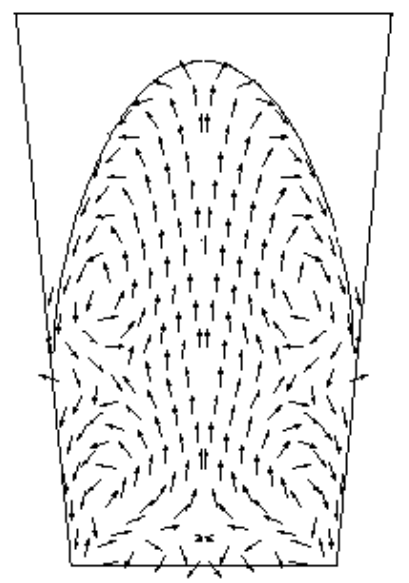

Figure 5: Velocity distribution.

\section{Conclusion}

The methodology described here is now used by the authors in many simulations concerning the influence of various inductor designs, supply parameters and crucible shapes on the process of induction heating and stirring of metals (Golak [4, 5]). Its main advantage, that is the fact that the deformation of the liquid metal surface is taken into account in calculations, offers new possibilities of a more precise optimisation of the devices for induction heating and stirring of liquid metals.

Knowing the shape of the free surface of the liquid metal and being able determine its actual size allow a more precise determination of these utility parameters of the devices that are somehow connected with the surface or generally with the shape of the molten metal. The most important of them are the power emitted in the charge and exchange of the elements between the liquid metal and the surrounding atmosphere. 
Our meta-system for the type of simulation described above consists of two commercial packages, which are (a) programme for electromagnetic calculations Cedrad 2D and (b) programme for hydrodynamic calculations Ansys Fluent. However, the presented methodology of triple coupling of electromagnetic field, flow field and liquid metal geometry supported by mathematical extrapolation of force field may be based on any software created for this kind of calculations.

\section{Acknowledgement}

This research work was carried out within project No. N508 034 31/1889, financially sponsored by the Polish Ministry of Science and Higher Education.

\section{References}

[1] Adler K., Schwarze, R.: Numerical Modelling of the Evaporation Process of an Electromagnetically Stirred Copper Melt, FLUENT CFD Forum 2005 Bad Nauheim, 2005

[2] Blacha L., Fornalczyk A., Przyłucki R., Golak S.: Kinetics of the evaporation process of the volatile component in induction stirred melts, 2nd International Conference Simulation and Modelling of Metallurgical Processes in Steelmaking STEELSIM 2007, Graz, Austria, pp. 389-395, 2007

[3] Hirt C. W., B. D. Nichols: Volume of Fluid (VOF) Method for the Dynamics of Free Boundaries. J. Comput. Phys., 39, pp. 201-225, 1981

[4] Golak S., Przyłucki R.: Oxidation of the surface of a liquid metal in the induction furnaces., Acta Metallurgica Slovaca 13, pp. 256-259, 2007

[5] Golak S., Przyłucki R.: The optimization of an inductor position for minimization of a liquid metal free surface, Electrotechnical Review, 11/2008, SIGMA-NOT, pp. 163-164, 2008 